\title{
A Simple Low-Cost Simulation Protocol for Approximate Localization of Structural Water Molecules in DNA Oligonucleotides
}

\author{
Amarílis de V. Finageiv Neder and Marçal de Oliveira Neto* \\ Instituto de Química, Universidade de Brasília, Campus Darcy Ribeiro, CP 04478, 70904-970 Brasília - DF, Brazil
}

Utilizando um protocolo combinado de mecânica e dinâmica molecular, de baixo custo computacional, empregando-se a expressão e os parâmetros do campo de força OPLS-AA, foi possível reproduzir as principais características da primeira camada de hidratação de heterooligonucleotídeos de DNA em duplas hélices na conformação A (1DPL) e B (1DPN e 1ENN), conforme descrições cristalográficas com resolução atômica; nosso protocolo também reproduziu satisfatoriamente as características das primeiras camadas de hidratação de homo-oligonucleotídeos de DNA na conformação B $\left[(\mathrm{AT})_{12}\right.$ e $\left.(\mathrm{CG})_{12}\right]$ obtidas em simulações por dinâmica molecular empregando-se protocolos mais longos e mais sofisticados. Um modelo preliminar da primeira camada de hidratação de oligonucleotídeos poderia ser útil para aqueles interessados em proceder cálculos mecânico-quânticos em sistemas cujas características de hidratação são desconhecidas em nível molecular ou, ainda, para refinamento de estruturas cristalográficas por comparação com padrões de difração experimentais.

Using a computational low-cost protocol by combining molecular mechanics energy minimization and molecular dynamics employing the OPLS-AA force field, we were able to reproduce the main structural features of the first hydration shell of double-helix DNA heterooligonucleotides in the A (1DPL) and B-conformations (1DPN and 1ENN), whose coordinates are available with atomic resolution from crystallographic data. Our simple protocol also reproduced the main hydration patterns of DNA homo-oligonucleotides in the B-conformation $\left[(\mathrm{AT})_{12}\right.$ and $(\mathrm{CG})_{12}$ ], obtained before by computer simulation using a longer and more sophisticated molecular dynamics protocol. A preliminary model of the first hydration shell of oligonucleotides may be very useful to those interested in performing quantum-mechanical calculations of systems where hydration features are unknown at the molecular level; the model may also be used by crystallographers during refinement steps.

Keywords: nucleic acids, hydration, quenched dynamics, bound water

\section{Introduction}

Water is a ubiquitous molecule in the biological environment and is considered to be an intrinsic component of the structure of nucleic acids (structural water molecules). Many ligands, such as amino acids, drugs or even other nucleotides, interact with nucleic acids either through a water bridge or by replacing structural water molecules bound to specific sites or, most commonly, by both types of contacts. ${ }^{1}$ Although the preferential hydration sites of nucleic bases are quite conservative according to comparative crystallographic data, ${ }^{2}$ different nucleotide sequences, ${ }^{3}$ base compositions, ${ }^{4}$ and conformations ${ }^{5}$ affect hydration in a very complex manner. In order to understand

\footnotetext{
* e-mail:marcal@unb.br
}

the function of this type of biomolecule in the cell environment or even inside cell organelles, it is important to investigate nucleic acid hydration sites more extensively, including the dynamics of the first hydration shells, the energetics of water binding, and the role of hydration and ions in nucleic acid conformations and ligand recognition-binding processes as well. To reach this goal, different complementary strategies in experimental and theoretical fields must be considered.

In early fiber diffraction studies it was shown that water content affects the DNA helix geometry, inducing a conformation transition from B to A-DNA at relatively low humidities between 75 and $80 \% .^{6}$ The first experimental studies about preferable hydration sites in DNA films as a function of relative humidity used IR spectroscopy techniques. $\mathrm{OH}$ vibrations different from those due to liquid 
$\mathrm{H}_{2} \mathrm{O}$ were observed and were attributed to the first hydration shell of water molecules interacting with DNA atoms with different relative strengths. ${ }^{6}$ The absorption frequencies of certain groups such as $\mathrm{P}-\mathrm{O}, \mathrm{P}-\mathrm{O}^{-}, \mathrm{C}=\mathrm{O}, \mathrm{C}-\mathrm{O}$, heterocyclic $\mathrm{N}$, $\mathrm{NH}$ and $\mathrm{NH}_{2}$, were also changed due to the effect of humidity. Another useful technique for studying DNA hydration and the location of water molecules is X-ray crystallography. There are a huge number of reviews concerning this subject and we will discuss only those results which were considered for the development of this work. One of the most studied deoxyribonucleotides has been the "Dickerson-Drew" (DD) dodecamer [d(CGCGAATTCGCG) ${ }_{2}$ ], a double-helix DNA with B conformation in which a total of 72 localized water molecules and a "spine of hydration" were first observed in the minor groove of the center of the AATT tract; hydration of major groove $\mathrm{N}$ or $\mathrm{O}$ atom bases was predominantly monodentated and the phosphate groups were the most hydrated sites. ${ }^{7}$ Today, atomic resolution X-ray data describe regular water networks and bridges of hydrogen-bonded waters around DNA oligomers, whose characteristics seem to be conformation-dependent. ${ }^{5}$ Due to the high quality of some of these reported crystallographic data, in terms of the description of hydration sites, they can be considered as reliable experimental standards for supporting theoretical approaches. ${ }^{5,8}$

Over the past few years, advances in computer simulation have prompted researchers to study the dynamics of water molecules and ions explicitly represented around DNA oligonucleotides comprising from 10 to 28 base-pairs. ${ }^{9}$ However, depending on the chosen program, the protocol or the level of theory, such calculations are time-consuming and require a high computing power. Here we present a simple low-cost protocol involving mixed steps of molecular dynamics (MD) and energy minimization (EM) calculations which can reproduce the main features of the hydration profile of nucleic acid oligomers as described by crystallographic studies. A simpler procedure than that given here, which also involves combined steps of EM and MD, was successfully used to describe a hydration model for the antiepileptic drug vigabatrin and the prosthetic group interaction. ${ }^{10}$ The current protocol based on "quenched dynamics" methodology was performed with explicitly represented water molecules (TIP3P), ${ }^{11}$ with free vacuum boundaries, without counter-ions in a total $20 \mathrm{ps}$ simulation using the OPLS-AA force field ${ }^{12}$ implemented in the Hyperchem program. ${ }^{13}$ Validation of the methodology was carried out by comparing our results to the high-resolution crystallographic data of two DNA heteroligomers in the $\mathrm{B}$ conformation (PDB codes: $1 \mathrm{DPN}^{5}$ and $1 \mathrm{ENN}^{8}$ ), a DNA decamer in the A-conformation (PDB code: $1 \mathrm{DPL}^{5}$ ), and two homoligomers $\mathrm{d}(\mathrm{CG})_{12}$ and $\mathrm{d}(\mathrm{AT})_{12}$, whose first shell hydration characteristics were described by Auffinger and Westhof ${ }^{14}$ using a much more sophisticated MD protocol and longer simulation ( 10$12 \mathrm{~ns}$ ). With this simple protocol one may quickly produce a reliable model of the main hydration patterns with the approximate localizations of structural water molecules of an oligonucleotide as would be found in its crystal structure. Such a preliminary model may be very useful to those interested in performing refined quantum-mechanical calculation of hydrated oligonucleotides whose first hydration shell is not known at the molecular level; it can also be used by crystallographers in order to prepare the first hydration model of a nucleic acid oligomer to be refined according to the experimental diffraction pattern.

\section{Experimental}

\section{Model systems}

The high quality X-ray crystallographic analysis of the A-DNA decamer [d(GCGTA ${ }^{\mathrm{M}}$ TACGC $)_{2}$ ], and the B-DNA dodecamer [d(CGCGAA $\left.{ }^{\mathrm{F} T T C G C G}\right)_{2}$ ], at 0.83 and $0.95 \AA$, respectively, by Egli and co-workers, ${ }^{5}$ is so fully described that we considered their results to be one of the best experimental data published to date for the purpose of validation of computationally simulated hydration sites of double-helical DNAs.

For simulation, all DNA double helix oligomers studied (see Table 1) were drawn using the database/ building modules of Hyperchem, except in the case of B-

Table 1. General description of double-helix DNA oligomers used as reference to validate our protocol

\begin{tabular}{|c|c|c|c|c|c|}
\hline Our DNA Denominations ${ }^{a}$ & Reference Sequences & Techniques & Resolution $(\AA)$ & PDB Code & Ref. \\
\hline B-DNA-DD & CGCGAA $^{\mathrm{F} T T C G C G}{ }^{\mathrm{b}}$ & X-rays & 0.95 & $1 \mathrm{DPN}$ & 5 \\
\hline A-DNA & GCGTA $^{\mathrm{M}} \mathrm{TACGC}^{\mathrm{b}}$ & $\mathrm{X}$-rays & 0.83 & 1DPL & 5 \\
\hline B-DNA-López & GCGAATTCG & $\mathrm{X}$-rays & 0.89 & $1 \mathrm{ENN}$ & 8 \\
\hline B-DNA-(TpA) 12 & TATATATATATA & MD & - & - & 13 \\
\hline B-DNA- $(\mathrm{CpG})_{12}$ & CGCGCGCGCGCG & MD & - & - & 13 \\
\hline
\end{tabular}

aThe letters A and B refer to the helix conformation, DD refers to Dickerson-Drew sequence, and López to the last name of the first author of 1ENN descriptive paper; ${ }^{\mathrm{b}} \mathrm{F} \mathrm{T}$ stands for 2'-deoxy-2'-fluoroarabino-thymine and ${ }^{\mathrm{M}} \mathrm{T}$ stands for 2'-methoxy-3'-methylene-phosphonate-thymine. 
DNA-López, where the crystallographic coordinates were directly extracted from PDB (crystallization water molecules and ions were excluded).

Since 1DPL and 1DPN crystallographic coordinates were fragmented, B-DNA-DD and A-DNA sequences were drawn without modifications on the thymidine backbone, which were introduced to facilitate crystallization (root mean-square atomic deviation, RMSD, between 1DPN and 1DPL and our draws were not higher than $0.46 \AA$ ). B-DNADD has the same sequence as the Dickerson-Drew dodecamer, which contains the recognition site of EcoRI restriction enzyme. DNA phosphate groups were kept charged and the phosphodiester oxygens O3' and O5' in the phosphate groups at the helix extremities were manually saturated with hydrogens.

In the case of B-DNA-López, the hydrogens of O3' from G9, and O1P and O2P from G1 at 5' were manually added while the remaining hydrogens were automatically attributed by Hyperchem. Only in this system, the coordinates of all non-hydrogen atoms were held fixed while the geometry of hydrogens was optimized in vacuum using the Polak-Ribière conjugate gradient method with OPLS-AA force field up to a RMS gradient convergence of $0.01 \mathrm{kcal} / \AA \mathrm{mol}$.

The DNA double helix was surrounded by a cubic box of about 1000 to 1300 TIP3P water molecules corresponding to a hydration shell with 10 to $15 \AA$ thickness (Table 2). In Hyperchem ${ }^{13}$ these water molecules come from a cubic water box comprising 216 molecules previously equilibrated according to a procedure developed by Jorgensen and co-workers using the Monte Carlo method. When the system encompasses more than 216 water molecules, several identical copies of the box are merged in a larger box to create a new box of $3 \times 3 \times 3 \times 216$ water molecules, from which the solvent water molecules are extracted. Since the extra water molecules are images of the original Jorgensen box, it is necessary to perform an initial EM calculation with these water molecules prior to the first MD step. No periodic boundary condition was used, and MD simulations were carried out as if each DNA double-helix was imbibed in a water drop surrounded by vacuum. No counter-ions were added since Egli and co-workers ${ }^{5}$ did not report a significant number of ions in the first hydration shell of 1DPL and 1DPN deoxyribonucleotides; also, according to Mazur, ${ }^{15}$ charge neutrality is not required for MD simulations of DNA chains in the case of an isolated cluster like this one.

\section{Methods and simulation protocol}

All calculation procedures were carried out using the OPLS-AA force field ${ }^{12}$ as implemented in Hyperchem 6 Professional. ${ }^{13}$ This program was run in a PC (Pentium III $900 \mathrm{MHz}$ and Pentium IV 1.6 GHz, 256Mb RAM) hardware system. The Polak-Ribière conjugate gradient method was chosen to perform all EM calculations up to an RMS gradient convergence of $0.01 \mathrm{kcal} / \AA ̊$ mol. Since water was explicitly represented, the dielectric constant $\varepsilon=1$ was set; inner and outer cutoffs, with switching function, were automatically assigned by the program as half of the smallest dimension of the initial water box (outer cutoff) minus $4 \AA$ (inner cutoff). During both MD runs, a time increment (stepsize) of $1 \mathrm{fs}$ was selected. No special strategy or algorithm was employed to calculate long-range electrostatic interactions - the Coulombic term of OPLSAA force field ${ }^{12}$ was used.

Keeping atomic coordinates of DNAs fixed during the whole protocol, the following five steps were successively carried out with the water molecules only: 1) EM; 2) MD at $800 \mathrm{~K}$ during $5 \mathrm{ps}$; 3) EM of the last geometry; 4) MD at $300 \mathrm{~K}$ during 15 ps using a random initial velocity; 5) EM of the last geometry. Whenever necessary, after the second step any water molecule which "evaporated" beyond the external boundary at a distance long enough to forbid a hydrogen bond (H-bond) contact to water molecules of the main water cluster was manually deleted.

The "quenched dynamics" method was chosen as a computational resource to improve EM procedures. Performing an MD simulation at high temperatures is an artificial tool to force the system to cross possible energy barriers.

Although OPLS-AA force field ${ }^{12}$ was especially developed to simulate hydrated nucleic acids, to our knowledge there is no paper in the literature concerning the hydration of nucleic acid oligomers by MD using this force field.

Table 2. Initial parameters of the DNA model systems

\begin{tabular}{llll}
\hline DNAs & Water Box Dimensions $\left(\AA^{3}\right)$ & Inner/outer cutoffs $(\AA)$ & Total Water Molecules \\
\hline B-DNA-DD & $31 \times 29 \times 51$ & $10.5 / 14.5$ & 1209 \\
A-DNA & $30 \times 30 \times 42$ & $11 / 15$ & 1012 \\
B-DNA-López & $28 \times 30 \times 43$ & $10 / 14$ & 956 \\
B-DNA-d(TpA) $)_{12}$ & $32 \times 29 \times 51$ & $10.5 / 14.5$ & 1269 \\
B-DNA-d(CpG) & $32 \times 29 \times 52$ & $10.5 / 14.5$ & 1308 \\
\hline
\end{tabular}




\section{Analytical procedures}

The localization of preferable hydration sites was done by visual inspection after the final EM step. The H-bonds were automatically assigned by Hyperchem with dotted lines according to a geometric criterion where the distance between the acceptor atom ( $\mathrm{N}$ or $\mathrm{O}$ ) and the hydrogen from the donating group ( $\mathrm{H}$ bonded to $\mathrm{N}$ or $\mathrm{O}$ ) is $\mathrm{d} 3.2 \AA$ and the angle between covalently bounded atoms and the acceptor atom is $>150^{\circ}$. The visual inspection method consisted of counting the number of water molecules which were interacting through $\mathrm{H}$-bonds with nucleic acid atoms and identifying some typical hydration patterns which permit the distinction between DNA oligomers in the A and Bconformations, and homo- and heteroligomers.

Our quali-quantitative results were compared with atomic resolution crystallographic data-1DPL, 1DPN and $1 \mathrm{ENN}$ in order to validate the protocol. In the case of the two homoligomers (B-DNA-d(TpA) ${ }_{12}$ and B-DNA$\left.\mathrm{d}(\mathrm{CpG})_{12}\right)$, the results were compared with those described by Auffinger and Westhof, ${ }^{14}$ who simulated the hydration of these double-helix B-DNAs using a different force field and a more sophisticated protocol than ours.

An approximate radial distribution of water molecules was obtained using the DETPDB program, ${ }^{16}$ although this program was not developed to calculate a radial distribution function. By selecting a part or the whole number of atoms of the solute, DETPDB builds a file with all atoms at a pre-specified distance from the selected solute. For the purpose of counting the total number of water molecules around the solute at certain distances, the number of water oxygen atoms was always considered. By employing DETPDB, we also estimated the relative density of groups of water molecules inside the volume of radial shells around DNA at different distances - we assumed that the DNA was condensed at the center of a spherical radial distribution of water molecules; the distance from the boundary of each radial slice to the DNA was considered as the radius ( $\mathrm{r}$ ) of the sphere and the volume (v) was calculated using $v=4 / 3 \pi r^{3}$. From the number of water molecules and the corresponding mass, the density of each slice was obtained in $\mathrm{g} / \mathrm{cm}^{3}$.

\section{Results and Discussion}

For each system the protocol was repeated at least twice, but no great discrepancies were seen in the quantitative data, including energy values and relative number of water molecules interacting directly with nucleic acid atoms. As was expected from a qualitative point of view, in each simulation a different snapshot from the hydration profile was caught but, surprisingly, the main hydration patterns, which can distinguish an A-DNA from a B-DNA, or B-DNA homo and heteroligomers, were maintained.

\section{$B-D N A-D D$}

Based on visual inspection, a higher hydration (number of the total water molecules interacting through H-bond according to Hyperchem definition - see Table 3) was observed: 1) on the phosphate groups in relation to other hydrophilic groups; 2) on oxygen $\mathrm{O}^{2}$ ' in relation to O5'; 3 ) on major groove base atoms in relation to minor groove base atoms. These observations are in accordance with the atomic resolution crystallographic data of the DNA dodecamer 1DPN. ${ }^{5}$ The main atoms involved in hydration according to our model are also the same as those observed in the X-ray study, namely the major groove atoms $\mathrm{N} 7(\mathrm{G})$, N7(A), N6(G), N6(A), N4(C) and O4(T) and the minor groove atoms $\mathrm{O} 2(\mathrm{~T})$ and $\mathrm{N} 3(\mathrm{~A})$; the hydration of minor groove atoms from the terminal CG base pairs O2(C), N2(G) and N3(G) was almost completely absent in our simulations. One of the few discrepancies between our results and those published by Egli and co-workers ${ }^{5}$ is the excessive hydration of phosphodiester oxygens O3' observed in the simulation, probably due to force field parameterization.

The main hydration patterns observed during our simulation are the highly hydrated phosphate groups, with 8 to 9 water molecules per nucleotide pair, and adjacent phosphate oxygens $\mathrm{O} 1 \mathrm{P}$ and $\mathrm{O} 2 \mathrm{P}$ interconnected by water bridges formed by two, three or more molecules.

As pointed out by Egli and co-workers, ${ }^{5}$ we also observed the solvent acting as a network that fills the helix grooves, creating a web around the DNA and, as part of this network, forming some ordered structures such as the well-known spine of hydration and ribbons of fused water hexagons and pentagons. Since it was first observed by Drew and Dickerson, ${ }^{7}$ the spine of hydration has been described as a string of water molecules that interconnect non-base-paired adjacent adenines and thymines from opposite strands at the floor of the minor groove. This spine, which is particularly regular in the AATT tract, bridges minor groove atoms $\mathrm{N} 3(\mathrm{~A})$ and $\mathrm{O} 2(\mathrm{~T})$ and, at the central ApT step, connects thymines $\mathrm{O} 2$ from different strands. In our simulations it was possible to identify these hydration patterns, even though they came from a different criterion (Hyperchem H-bond assignment) which was not based on the water center of mass, as is usually reported in experimental work. In agreement with the crystallographic study of $1 \mathrm{DPN},{ }^{5}$ we observed the recurrent participation of oxygen $\mathrm{O} 4$ ' in the spine motives. Figure 1 shows an example 
of a hydration spine observed in our simulations and Figure 2 shows some interesting polygonal structures extracted from the water network.

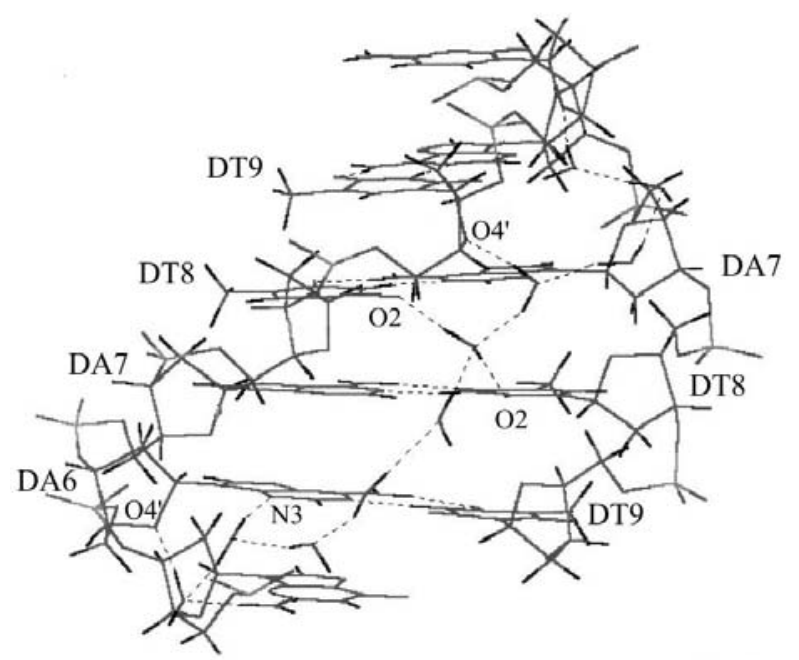

Figure 1. A spine motive seen in AATT tract interconnecting adenines and thymines non-base-paired from opposite strands, involving minor groove atoms N3(A), O2(T) and O4' (from B-DNA-DD simulations).

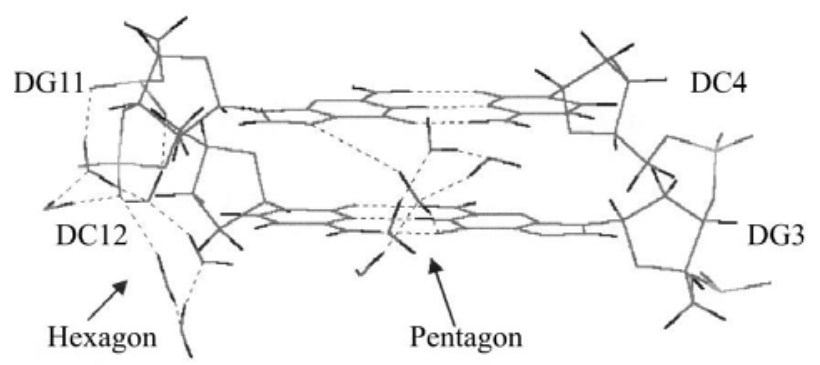

Figure 2. Hexagonal and pentagonal water arrangements observed in the minor groove CG portion (from B-DNA-DD simulations).

\section{$A-D N A$}

As shown in Table 3, of all of the potentially hydrophilic groups of the A-DNA decamer, the phosphate groups and the oxygen $\mathrm{O}^{\prime}$ ' were the most hydrated. The average number of water molecules interacting through $\mathrm{H}$-bond with ribose $\mathrm{O} 4$ ' and atoms of the nucleic bases were comparable. These observations are in agreement with the crystallographic data described for 1DPL by Egli and co-workers. ${ }^{5}$ Concerning the nucleic bases, in our simulations at least one water molecule was found interacting through a $\mathrm{H}$-bond with major groove atoms N7(G), N7(A), O6(G), N4(C), and O4(T), and also with minor groove atoms $\mathrm{O} 2(\mathrm{~T})$ and $\mathrm{N} 3(\mathrm{~A})$. In contrast to the experimental work, ${ }^{5}$ the simultaneous hydration of all these nucleic base atoms was not observed, probably due to differences between the geometric criteria adopted in selecting the water molecules of the first hydration shell.

Due to a more compact conformation, adjacent A-DNA phosphate groups were interconnected by only one or two water molecules, as described for 1DPL. ${ }^{5}$

In the case of 1DPL, Egli and co-workers ${ }^{5}$ did not observe water molecules at distances shorter than $3.6 \AA$ to nitrogen N6 from the adenine exocyclic amino group, which prefers to interact with oxygen $\mathrm{O} 4$ in stacked thymines in the same strand. The DNA conformational constraint imposed by our protocol and the parameterization of OPLS-AA force field allowed the hydration of this exocyclic amino group in the A-DNA decamer, and a water bridge interconnecting the adenine N6 group and the carbonyl oxygen $\mathrm{O} 4$ of the adjacent thymine was observed.

Also, with $1 \mathrm{DPL}^{5}$ it was observed that at the periphery of the major groove, fused hexagons and pentagons of water molecules connected phosphate groups with the base atoms. This hydration pattern was also observed in our simulation. As described for B-DNA-DD, the A-DNA decamer presents a huge water network that fills all grooves and involves the whole DNA, but no regular arrangement is seen in the major grooves, except for the abovementioned fused polygons.

\section{$B-D N A-D D$ versus A-DNA}

The reasons why a double helix prefers an A or B-conformation is still a matter for discussion. Nevertheless, some experimental evidence suggests that in low humidity environments the more compact Aconformation is favored over the B-conformation. ${ }^{17}$ The more modest hydration of the 1DPL phosphate groups compared with 1DPN, due to the compactness that allows just one or two water molecules to bridge adjacent phosphate oxygens, was remarked upon by Egli and coworkers. ${ }^{5}$ As shown in Table 3, our simulations reproduced this behavior, as well as the more extensive hydration of O1P in B-DNA-DD in comparison with A-DNA. As reported for 1DPL and 1DPN, ${ }^{5}$ we also observed that oxygens O3' are more hydrated in A-DNA than in B-DNA-DD.

In the simulations of B-DNA-DD and A-DNA, all potentially hydrophilic atoms of the terminal CG pairs interact with water molecules in contrast to the crystallographic study, where minor groove atoms $\mathrm{O} 2(\mathrm{C})$, $\mathrm{N} 2(\mathrm{G})$ and $\mathrm{N} 3(\mathrm{G})$ interact mostly with DNA atoms from neighboring helices.

Feig and Pettitt ${ }^{17}$ compared the hydration profile of DNA oligomers in the A and B conformations obtained by long-duration ( 10 ns) molecular dynamics simulations 
Table 3. Average number of water molecules (W) interacting through H-bond with hydrophilic nucleic acids atoms in the A-DNA decamer and B-DNA-DD dodecamer simulations, and the average number of water molecules per base pair in each sequence

\begin{tabular}{|c|c|c|c|c|c|c|c|c|}
\hline Simulation & $\begin{array}{l}\text { Average Total } \\
\text { Number of } \mathrm{W}\end{array}$ & $\mathrm{O} 1 \mathrm{P} / \mathrm{O} 2 \mathrm{P}$ & O1P & O3' & O5 & $\mathrm{O} 4^{\prime}$ & $\begin{array}{c}\text { "Major Groove" } \\
\text { Base Atoms }\end{array}$ & $\begin{array}{c}\text { "Minor Groove" } \\
\text { Base Atoms }\end{array}$ \\
\hline A-DNA & 142.5 & 77 & 43.5 & 19 & 4.5 & 12 & 14.5 & 15.5 \\
\hline A-DNA/10 base pairs & 14.3 & 7.7 & 4.4 & 1.9 & 0.5 & 1.2 & 1.5 & 1.6 \\
\hline B-DNA-DD & 145.5 & 102 & 56.5 & 12 & 1.5 & 8.5 & 14 & 7.5 \\
\hline B-DNA-DD/12 base pairs & 12.1 & 8.5 & 4.7 & 1 & 0.1 & 0.7 & 1.2 & 0.6 \\
\hline
\end{tabular}

with experimental data. As noted by the authors, MD simulations usually observe the individual hydration of the guanine exocyclic amino group -N2(G), although the average water densities near the $\mathrm{N} 2$ atom calculated by Schneider and co-workers ${ }^{2}$ indicated that in B-DNAs N2(G) is almost completely unhydrated, while $\mathrm{N} 3(\mathrm{G})$ is the most hydrated site in the minor groove; in the case of A-DNAs, the highest water density is located between N2(G) and N3(G). Feig and Pettitt ${ }^{18}$ investigated this issue using only high-resolution crystal structures to compare with their simulated densities, and concluded that the individual $\mathrm{N} 2(\mathrm{G})$ hydration is statistically plausible, supporting the results obtained in our simulations.

Another observation from Feig and Pettitt's ${ }^{18}$ MD simulations which was not reported in experimental studies and differentiates DNA oligomers in the A- and Bconformations is the presence of water bridges interconnecting, in the same strand, the minor groove atoms $\mathrm{N} 3$ or O2, mainly in the AT tract, with ribose O4' of the adjacent sugar, a characteristic seen only in A-DNA simulations. In our simulations, this hydration pattern was also more often verified in the A-DNA system.

\section{B-DNA-López}

The description of the arrangements of the water molecules of the first hydration shell of DNA nonamer $1 \mathrm{ENN}^{8}$ is rather limited, except for the hydration spine at the central AATT sequence. López and co-workers ${ }^{8}$ gave the total number of water molecules which are in contact with one or more DNA atoms (97) and remarked that, in general, the hydration sites agreed with those described by Schneider and co-workers. ${ }^{2}$ They also highlighted that most phosphate groups have four to five associated water molecules and that polygons of different sizes are the main structures of the water network that covers the whole duplex. ${ }^{8}$

In our simulations of B-DNA-López we observed a similar hydration picture, as can be confirmed in Table 5 . The phosphate oxygens were the most hydrated sites in the double-helix and, on the average, four water molecules were found interacting with each phosphate group.
Although not described by López and co-workers, we also observed interphosphate water bridges formed by two, three or more water molecules as was obtained in the B-DNADD simulations, predominantly at the central AT sequence in both strands. In B-DNA-López simulations polygonal structures were recognized in the water network involving the duplex, and a water spine motive was also noticed, with the notable participation of the ribose O4' and the minor groove atoms of the AT tract.

\section{Radial distribution of water molecules and water density}

The definition of hydration shells in experimental studies depends on the technique employed. In structural analysis by X-ray diffraction, the spatial ordering of the water molecules and the B-factor reflects the differences among hydration shells. Egli and co-workers ${ }^{5}$ defined the first hydration shell of 1DPL and 1DPN as formed by water molecules interacting with the $\mathrm{N}$ and $\mathrm{O}$ nucleic acid atoms at a distance (d) range of $\mathrm{d} d 3 \AA$ and $3.0 \AA<\mathrm{d}<3.3 \AA$, and, for the second hydration shell, at $3.3 \AA<\mathrm{d}$ d $3.6 \AA$. The total number of water molecules found in the first and second hydration shell of $1 \mathrm{DPL}^{5}$ was 127 and 42, respectively, and for $1 \mathrm{DPN}^{5} 150$ and 72 . For $1 \mathrm{ENN},{ }^{8}$ even if no attempt was made to identify hydration layers, it was reported that 97 from a total of 151 water molecules were interacting with one or more DNA atoms. Although theoretical studies can intuitively find the correspondence between the arrangements of hydration patterns and solvation shells, the calculation of a radial distribution function and the identification of minima in the curve are usually applied to define solvation shells around DNA. Feig and Pettitt ${ }^{18}$ used this criterion to determine the hydration layers around the DNA decamer $d\left(\mathrm{C}_{5} \mathrm{~T}_{5}\right) \cdot\left(\mathrm{A}_{5} \mathrm{G}_{5}\right)$ after a 10-12ns MD simulation, estimating the boundaries of the first and second hydration shell to the closest DNA atom: $3.5 \AA$ and $6.1 \AA$ from oxygen, $3.9 \AA$ and $6.3 \AA$ from nitrogen, and $5.2 \AA$ and $7.5 \AA$ from carbon.

The radial distribution of water molecules in our simulations was estimated approximately using the program DETPDB ${ }^{16}$ and we observed similar results for BDNA-DD, A-DNA and B-DNA-López: in all cases the first 
Table 4. Comparison of the approximate average number of first shell water molecules for all simulations studied obtained by visual inspection and by the DETPDB program with crystallographic data for the DNA oligomers used as references in our studies

\begin{tabular}{|c|c|c|c|c|c|}
\hline \multirow{3}{*}{ Simulations } & \multicolumn{5}{|c|}{ Approximate number of first shell water molecules } \\
\hline & \multirow{2}{*}{$\begin{array}{l}\text { by Visual } \\
\text { Inspection }\end{array}$} & \multirow{2}{*}{$\begin{array}{c}\text { by DETPDB } \\
\text { Distance range from } \\
\text { DNA } 2.5 \text { to } 3.0 \AA\end{array}$} & \multicolumn{3}{|c|}{ Crystallographic Data } \\
\hline & & & $1 \mathrm{DPN}^{5}$ & $1 \mathrm{DPL}^{5}$ & $1 \mathrm{ENN}^{8}$ \\
\hline B-DNA-DD & 145.5 & 148 & 150 & & \\
\hline A-DNA & 132.5 & 133.5 & & 127 & \\
\hline B-DNA-López & 102 & 117.5 & & & 97 \\
\hline $\mathrm{d}(\mathrm{TpA})_{12}$ & 145 & 164 & & & \\
\hline $\mathrm{d}(\mathrm{CpG})_{12}$ & 153 & 163 & & & \\
\hline
\end{tabular}

maximum in the radial distribution graphic is between 2.5 and $3.0 \AA$ and the following minimum is between 3.3 and 3.6-4.0 $\AA$, numbers that are in agreement with the theoretical ${ }^{17}$ and experimental definitions ${ }^{5}$ of the first hydration shell of DNA oligomers given above. In all simulations, the total number of water molecules interacting with the nucleic acid atoms through $\mathrm{H}$-bonds, according to the visual inspection method, was comparable to the number of water molecules of the supposed first hydration shell using the DETPDB program. ${ }^{16}$ The total number of water molecules in the first layer in the simulations of B-DNA-DD, A-DNA or B-DNALópez is very close to the reported numbers for $1 \mathrm{DPN}^{5}$, $1 \mathrm{DPL}^{5}$, and $1 \mathrm{ENN}^{8}$ (Table 4).

Another way to perform the theoretical estimate of solvation layers is by the approximate determination of the relative density of a group of water molecules at regular distances from the DNA molecule. As explained in the description of analytical procedures (see Experimental Section), in all simulated systems the distance range related to the maximum in the density graph coincided with the first maximum in the radial distribution graph between 2.5 and $3.0 \AA$ (see Figure 3). Apparently, our protocol is able to satisfactorily delimit the first hydration layer of a

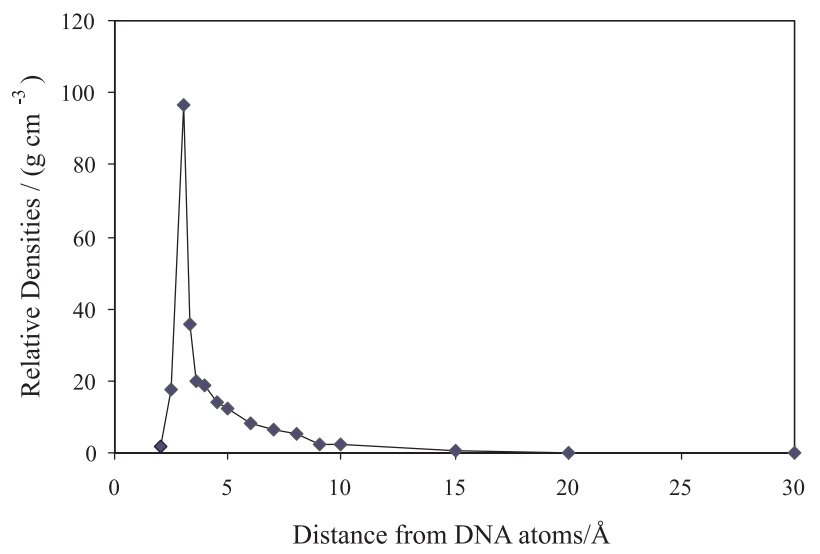

Figure 3. Example of the relative densities profile obtained for one of the simulations of B-DNA-DD.
DNA double-helix oligomer comprising from 9 to 12 pairs of nucleotides.

$B-D N A-d(T p A)_{12}$ versus $B-D N A-d(C p G)_{12}$

The total and average number of water molecules per functional group obtained after the simulations of B-DNA$\mathrm{d}(\mathrm{AT})_{12}$ and B-DNA-d(CG) ${ }_{12}$ were counted by visual inspection. As shown in Table 5, superior hydration of the $\mathrm{O} 1 \mathrm{P}$ and $\mathrm{O} 2 \mathrm{P}$ phosphate oxygens in relation to the other functional groups in both simulations was observed; the hydration of the O3' and O5' phosphodiester oxygens is also comparable in the two systems, but the hydration of the ribose O4' oxygen and base atoms is almost $40 \%$ higher in the $\mathrm{d}(\mathrm{CG})_{12}$ double-helix. On the average, we found 12.8 water molecules interacting through $\mathrm{H}$-bonds, according to the Hyperchem criterion, with the $\mathrm{CG}$ pair of nucleotides $(\mathrm{C}\{\mathrm{G})$, and 12.1 with the AT pair of nucleotides $(A=T)$. Considering only the base atoms, we observed on the average 2.8 and 1.8 water molecules forming H-bonds with the CG and AT base-pairs, respectively. In relative terms, these results are in agreement with those obtained by Auffinger and Westhof, ${ }^{14}$ since they also found a slightly higher number of solvent species interacting with the $\mathrm{C}\{\mathrm{G}$ pairs than $\mathrm{A}=\mathrm{T}$ pairs, even when also taking into account the number of ions: 20.1 for $\mathrm{C}\{\mathrm{G}, 5.5$ for the $\mathrm{CG}$ base-pair, 19.8 for A=T, 5.1 for the AT base-pair; considering only the water molecules, they counted 5.2 for the CG base-pair and 5.0 for the AT base-pair. We attribute the differences in absolute values between our results and those of Auffinger and Westhof ${ }^{14}$ to the less restrictive geometric criteria they employed to assign the interacting solvent species.

In Auffinger and Westhof's simulations, ${ }^{14}$ most of the potentially hydrophilic sites in each pair of nucleotides were occupied by almost one water molecule. However, during the MD runs they noted that not all water molecules had the same potential to interact with DNA atoms through H-bonds; thus, they calculated hydrogen-bonding percentages $(\mathrm{HB} \%)$ defined as the total number of $\mathrm{H}$-bonds 
established between atoms of the DNA and the hydration shell during a single trajectory divided by the total number of configurations analyzed during the last $1.5 \mathrm{~ns}$ of simulation, considering an $\mathrm{H}$-bond criterion where the distance $\mathrm{d}(\mathrm{H} . . \mathrm{A})<2.5 \AA$ and the angle $\theta(\mathrm{D}-\mathrm{H} . . . \mathrm{A})>135^{\circ}$. Their results showed that $\mathrm{O} 1 \mathrm{P}, \mathrm{O} 2 \mathrm{P}$, major groove atoms $\mathrm{N} 7(\mathrm{G}$ and $\mathrm{A}), \mathrm{N} 4(\mathrm{C})$ and $\mathrm{O} 4(\mathrm{~T})$, and minor groove atoms $\mathrm{O} 2(\mathrm{C}$ and $\mathrm{T})$ and $\mathrm{N} 3(\mathrm{G}$ and $\mathrm{A})$ are apparently the strongest H-bond acceptors for solvent molecules, while O3', and especially O4' and O5', are weaker H-bond acceptors. The low $\mathrm{HB} \%$ for $\mathrm{O} 4$ ' was attributed to a supposed involvement of this atom in a non-conventional contact C2' - H...O 4', a peculiarity not seen in our simulations, probably due to the conformational constraint imposed on DNA or to another methodological reason. Similarly to Auffinger and Westhof's results, ${ }^{14}$ we did not record an expressive hydration of the exocyclic amine group N6 of adenines, and we also observed in the simulations of $\mathrm{d}(\mathrm{AT})_{12}$ and $\mathrm{d}(\mathrm{CG})_{12}$ a higher hydration of the phosphate oxygens O1P and $\mathrm{O} 2 \mathrm{P}$ and the base atoms N7(A and G), O4(T), O2(C and $\mathrm{T}), \mathrm{N} 4(\mathrm{C})$. In contrast, we noticed an appreciable hydration of atoms $\mathrm{O} 6(\mathrm{G})$ and $\mathrm{N} 2(\mathrm{G})$, and we did not observe a significant hydration of the minor groove N3(A and $\mathrm{G}$ ) - the hydration of the exocyclic amine group N2(G), close to the N3, was the preferable hydrophilic group. Concerning the carbonyl oxygen O6(G), Auffinger and Westhof ${ }^{14}$ found that this was one of the most important potassium ion $\left(\mathrm{K}^{+}\right)$interaction sites and in our simulation, without counter-ions, we observed a recurrent $\mathrm{H}$-bond interaction of $\mathrm{O6}(\mathrm{G})$ with water molecules.

A hydration pattern observed by Auffinger and Westhof ${ }^{14}$ only for $\mathrm{d}(\mathrm{CG})_{12}$ was the presence of long-lived water molecule bridges (W) interconnecting $\mathrm{O} 2(\mathrm{C})_{\mathrm{n}} \ldots \mathrm{W} \ldots \mathrm{O} 4{ }^{\prime}{ }_{\mathrm{n}+1}$ and $\mathrm{N} 3(\mathrm{G})_{\mathrm{n}} \ldots \mathrm{W} \ldots \mathrm{O} 4{ }^{\prime}{ }_{\mathrm{n}+1}$, which persisted for more than 150ps. In our simulation we also observed this type of water bridge only for $\mathrm{d}(\mathrm{CG})_{12}$, but in the case of guanine the bridge involved only the $\mathrm{N} 2$ atom, instead of N3.

During our simulations of B-DNA-d(CG) ${ }_{12}$ and B-DNA$\mathrm{d}(\mathrm{AT})_{12}$ we observed interphosphate bridges involving two or more water molecules, as already recorded for the BDNA-DD and B-DNA-López simulations.

In agreement with Auffinger and Westhof's simulations, ${ }^{14}$ we did not recognize the famous water spine connecting minor groove atoms in the simulation of BDNA-d(AT) ${ }_{12}$; the only observed hydration pattern was three isolated water bridges in different ApT steps linking the $\mathrm{O} 2$ oxygens from adjacent thymines of opposite strands, similar to the water bridge described in the AT tract of 1DPN.

During their simulations of DNA and RNA homoligomers, Auffinger and Westhof ${ }^{14}$ affirmed that "the good level of convergence obtained for these simulations is reflected by the similar number of water molecules calculated around the backbone atoms, a property which is non-sequence dependent". According to this criterion, we can also assume that our simulations achieved a good level of convergence if we compare the average number of water molecules interacting with the $\mathrm{O} 1 \mathrm{P}$ and $\mathrm{O} 2 \mathrm{P}$ phosphate oxygens (see Table 5).

\section{B-DNA homoligomers versus B-DNA heteroligomers}

Using high-precision densiometric and ultrasonic measurements, Chalikian and co-workers ${ }^{4}$ determined the apparent molar volumes and compressibilities of DNA oligomers with varying base compositions and base sequences, and reached some general conclusions about the hydration of B-DNA duplexes: the CG base-pairs are more hydrated than the AT base-pairs and mixed base sequences are less hydrated than either CG or AT B-DNA homopolymers. In agreement with this experimental study,

Table 5. Total number of water molecules per functional group observed during the simulations of B-DNA-d(AT) ${ }_{12}$ and B-DNA-d(CG) ${ }_{12}$, B-DNA-DD, B-DNA-López, A-DNA, and the average number of water molecules per pair of nucleotides and bases AT and CG

\begin{tabular}{|c|c|c|c|c|c|}
\hline \multirow{2}{*}{$\begin{array}{l}\text { Total Number and Average number of water molecules per } \\
\text { functional group counted by visual inspection }\end{array}$} & \multicolumn{4}{|c|}{ B-DNA } & \multirow[t]{2}{*}{ A-DNA } \\
\hline & $(\mathrm{CG})_{12}$ & $(\mathrm{AT})_{12}$ & $\mathrm{DD}$ & López & \\
\hline Total & 161 & 154 & 146 & 102 & 142.5 \\
\hline $\mathrm{O} 1 \mathrm{P}$ and $\mathrm{O} 2 \mathrm{P}$ & 97 & 107 & 102 & 69.5 & 77 \\
\hline $\mathrm{O}^{\prime}$ ' and $\mathrm{O} 5^{\prime}$ & 16 & 18 & 13.5 & 4.5 & 23.5 \\
\hline $\mathrm{O} 4$ & 14 & 8 & 8.5 & 8 & 12 \\
\hline "Minor groove" atoms & 18 & 11 & 8 & 9 & 15.5 \\
\hline "Major groove" atoms & 16 & 10 & 14 & 11 & 14.5 \\
\hline Average/O1P and $\mathrm{O} 2 \mathrm{P}$ & 4 & 4.5 & 4.25 & 3.9 & 3.9 \\
\hline Average/ CG nucleotides-pairs & 12.8 & - & 11.7 & 11.7 & 13 \\
\hline Average/ AT nucleotides-pairs & - & 12.1 & 11 & 10.2 & 13.7 \\
\hline Average/ CG bases-pairs & 2.8 & - & 1.8 & 2.5 & 3.3 \\
\hline Average/ AT bases-pairs & - & 1.8 & 2 & 1.4 & 2.7 \\
\hline
\end{tabular}


we observed in most studies, independently of base sequence or duplex conformation, the superior hydration of the CG base-pairs in relation to the AT base-pairs; our simulations also showed a higher hydration of the CG and AT pairs of nucleotides in homopolymeric B-DNAs in comparison to B-DNAs with mixed sequences, suggesting that our protocol is able to discriminate B-DNA homoligomers from B-DNA heteroligomers (see Table 5).

Selecting potentially hydrophilic sites in unknown structures

Some of the hydration patterns detected at the end of the simulation protocol were already present after the third step of our protocol. This observation suggested a simple criterion for selecting the most hydrophilic sites in molecules with unknown first shell hydration arrangements. According to this criterion, the sites that are hydrated from the third step of the protocol until the end of the simulation could be considered as potentially hydrophilic sites, and those water molecules that visit them and interact through $\mathrm{H}$-bonds, as assigned by Hyperchem, could eventually be considered as potential candidates for the crystallographic status of structural water molecules.

\section{Conclusions}

Using a simple low-cost computational protocol with mixed steps of molecular dynamics and energy minimization calculations employing the OPLS-AA force field, we were able to satisfactorily reproduce the main hydration patterns of the first shell water molecules of DNA heteroligomers in the A- and B-conformations, in agreement with atomic resolution crystal data. ${ }^{5,8}$

In the case of B-DNA homoligomers, the results obtained in our simulations were comparable to those described in the literature, which utilized a more sophisticated protocol and a longer MD simulation. ${ }^{14}$

Although our simulations were performed in the absence of counter-ions, without periodic boundary conditions and with a cut-off radius for the electrostatic calculations, we verified the principal structural arrangements of the water molecules of the first hydration layer, enabling the differentiation of the A-form from Bform DNA heteroligomers, and the B-DNA homoligomers from B-DNA heteroligomers as well.

Instead of average interaction distribution or location of water molecules of hydration, our protocol allows the approximate location of possible hydration sites including some water bridges, with similar characteristics to those described for high-resolution crystallographic structures.
The validity of such a protocol, which reproduces some of the hydration patterns around DNA oligomers seen in crystallographic studies, should be recognized, since longer and more sophisticated MD simulations that model the aqueous solution environment also correlate their results with crystallographic data. ${ }^{14,17-19}$ The agreement between the hydration profile of nucleic acids in solution simulations and in high-resolution crystal data supports the idea that crystal structures give a confident picture of the first hydration shell of DNA's oligomers, which may help in the understanding of many biologically relevant processes.

\section{Acknowledgements}

We thank Professor Dr. Bernard Maigret for providing us with the Hyperchem 6 Professional Package, and Professor Dr. Pedro G. Pascutti for the use of his DETPDB program. We also thank CAPES (PROCAD 003/01-8) and CNPq (Project 521071/01-1 (NV)) for support to this work. We are very grateful for relevant comments by the referees.

We are also thankful to Peter Bakuzis and Susie Casement Moreira for english revision and helpful comments during the preparation of this manuscript"

\section{References}

1. Halle, B.; Denisov, V. P.; Biopolymers 1998, 48, 210; Shotton, M. W.; Pope, L. H.; Forsyth, V. T.; Langan, P.; Grimm, H.; Rupprecht, A.; Denny, R. C.; Fuller, W.; Physica B (Amsterdam, Neth.) 1998, 241-243, 1166; Auffinger, P.; Westhof, E.; J. Mol. Biol. 2000, 300, 1113.

2. Schneider, B.; Cohen, D. M.; Schleifer, L.; Srinivasan, A. R.; Olson, W. K.; Bermas, H. M.; Biophys. J. 1993, 65, 2291.

3. Jacobson, A.; Leupin, W.; Liepinsh, E.; Otting, G.; Nucleic Acids Res. 1996, 24, 2911.

4. Chalikian, T. V.; Sarvazyan, A. P.; Plum, E. G.; Breslauer, K. J.; Biochemistry 1994, 33, 2394.

5. Egli, M.; Tereshko, V.; Teplova, M.; Minasov, G.; Joachimiak, A.; Sanishvili, R.; Weeks, C. M.; Miller, R.; Maier, M. A.; An, H.; Dan Cook, P.; Manoharan, M.; Biopolymers 1998, 48, 234.

6. Bloomfield, V. A.; Crothers, D. M.; Tinoco Jr, I.; Nucleic Acids - Structures, Properties, and Functions; University Science Books: California, 2000, ch. 11.

7. Drew, H. R.; Dickerson, R. E.; J. Mol. Biol. 1981, 151, 535.

8. López-Soler, M.; Malinina, L.; Subirana, J. A .; J. Biol. Chem. 2000, 275, 23034.

9. Cheatham III, T. E.; Young, M. A.; Biopolymers 2001, 56, 232; Beveridge, D. L.; McConnell, K. J.; Curr. Opin. Struct. Biol. 2000, 10, 182; Giudice, E.; Lavery, R.; Acc. Chem. Res. 2002, 35, 350 . 
10. Giambiagi, M.; Giambiagi, M.S.; Oliveira Neto, M.; Phys. Chem. Chem. Phys. 2001, 3, 5059.

11. Jorgensen, W. L.; Chandrasekhar, J.; Madura, J. D.; Impey, R. W.; Klein, M. L.; J. Chem. Phys. 1983, 79, 926.

12. Pranata, J.; Wierschke, S. G.; Jorgensen, W. L.; J. Am. Chem. Soc. 1991, 113, 2810.

13. HyperChem 6 Professional; Hypercube, Inc.: Florida, 2000.

14. Auffinger, P.; Westhof, E.; J. Mol. Biol. 2001, 305, 1057.

15. Mazur, A. K.; J. Am. Chem. Soc. 2002, 124, 14707.
16. Pascutti, P. G.; DETPDB, Program for Molecular Detail Display from $P D B$ Files, Universidade Federal do Rio de Janeiro, Brazil, 2001.

17. Feig, M.; Pettitt, B. M.; Biopolymers 1998, 48, 199.

18. Feig, M.; Pettitt, B.M.; J. Mol. Biol. 1999, 286, 1075.

19. Duan, Y.; Wilkosz, P.; Crowley, M.; Rosenberg, J. M.; J. Mol. Biol. 1997, 272, 553.

Received: April 1, 2004

Published on the web: March 15, 2005 